\title{
Matrix metalloproteinase-9 and p53 involved in chronic fluorosis induced blood-brain barrier damage and neurocyte changes
}

\author{
Shen Qing-Feng, Xia Ying-Peng, Xu Tian-Tong
}

Department of Spine Surgery, Tianjin Union Medical Center, Tianjin, China

Submitted: 13 October 2016

Accepted: 18 February 2017

Arch Med Sci 2019; 15, 2: 457-466

DOI: https://doi.org/10.5114/aoms.2019.83294

Copyright (@) 2019 Termedia \& Banach

\section{Abstract}

Introduction: A large number of basic and clinical studies have confirmed that fluoride produces toxic effects on multiple organ systems in the body including the nervous system.

Material and methods: One hundred twenty Wistar rats were randomly divided into 4 groups with 30 in each group: a high fluoride group (drinking $200 \mathrm{mg} / \mathrm{l}$ fluoridated water, 24 weeks); a high fluoride control group (drinking distilled water, 24 weeks); a fluoride removal group (drinking fluoridated water, $12 \mathrm{~W}$; then distilled water, $12 \mathrm{~W}$ ) and a defluorination control group (drinking distilled water, 24 weeks).

Results: The high fluoride and fluoride removal groups had spinal cord astrocyte edema. The apoptosis rate of spinal nerve cells in the high fluoride group and fluoride removal group were significantly higher $(p<0.01)$ than in the fluoride control and defluorination control group. The Evans blue (EB) content, matrix metalloproteinase-9 (MMP-9) and p53 expression in the high fluoride group and fluoride removal group were higher $(p<0.01)$ than in the fluoride control and defluorination control group.

Conclusions: The apoptosis of spinal cord nerve cells is obviously higher in rats with chronic fluoride exposure. Chronic fluoride exposure leads to high expression of MMP-9, and results in increased damage of the blood-spinal cord barrier. Increased p53 may be one of the factors causing damage. Shortterm removal of fluoride has no obvious recovery in apoptosis of spinal cord nerve cells; highly expressed MMP-9 and p53 may be one of the reasons for unrecovered function.

Key words: fluorosis, blood-brain barrier, nerve cells, rats, matrix metalloproteinase-9, p53.

\section{Introduction}

Fluorine is one of the essential trace elements for the life activities of the human body, and a low dose of fluoride has a positive effect in prevention of dental caries and treatment of osteoporosis [1]; but the safety range of fluoride in the human body is less than that of other trace elements [2]. Endemic fluorosis is a biogeochemical disease that occurs in a specific geographical environment. In addition to causing dental fluorosis and skeletal fluorosis, fluoride also induces multiple organ and tissue pathological damage $[3,4]$. The history of study of fluoride on human health has lasted over 80 years, and the specific mechanism is still under study. A large number of basic and clinical studies have confirmed that

\author{
Corresponding author: \\ Dr. Xia Ying-Peng \\ Department of Spine Surgery \\ Tianjin Union Medical Center \\ 190 Jieyuan Road \\ Tianjin 300121, China \\ Phone: +86 2287729595 \\ Fax: +862287729595 \\ E-mail: \\ yingpengxia5530@163.com
}


fluoride produces toxic effects on multiple organ systems in the body [5-8]. Researchers have paid more and more attention to the fluoride toxicity to the nervous system in recent years [9-11].

Fluoride can enter the brain through the bloodbrain barrier. Long-term intake of fluoride can cause the retention of excessive fluoride in the brain, and affect the normal physiological function of brain cells. In addition, fluoride can accumulate in the fetal brain tissue through the placental barrier [12]; the fluoride exerts its toxic effect on development and differentiation of nerve cells of the offspring brain, and leads to mental disorders of offspring. Recent studies have shown that fluoride intake is significantly correlated with children's intelligence $[13,14]$. A few studies have indicated the effect of chronic fluorosis on spinal nerves. Sun et al. [15] reported that chronic fluorosis could cause damage of the blood-brain barrier. However, whether excessive fluoride also has an obvious destructive effect on the blood-spinal cord barrier was not mentioned. Studies have shown that chronic fluorosis can cause elevated oxidative stress in the body or in vitro cell culture, resulting in apoptosis of cells [16, 17]. Increased permeability of the blood-brain barrier leads to a variety of neurological injuries [18]. The increased expression of matrix metalloproteinase-9 (MMP-9) is related to increasing permeability of the blood-brain barrier [19]. p53 is one of the main genes regulating signal transduction of cell apoptosis [20].

In this study, we observed the poisoning effect of chronic fluoride on blood-spinal cord barrier, in order to reveal the effect of fluorosis on blood-spinal cord barrier of rats, and investigated its possible mechanism.

\section{Material and methods}

\section{Animal and model preparation}

120 Wistar rats aged 1 month were randomly divided into 4 groups by a random number table, each group had 30 rats: a high fluoride group (drinking $200 \mathrm{mg} / \mathrm{l}$ fluoridated water, 24 weeks); a high fluoride control group (drinking distilled water, 24 weeks); a fluoride removal group (drinking fluoridated water, $12 \mathrm{~W}$; then distilled water, $12 \mathrm{~W}$ ) and a defluorination control group (drinking distilled water, 24 weeks). All animal experiments were performed under approved protocols of the institutional animal use and care committee.

The 24-hour urine at weeks 4, 8 and 12 were collected by stainless steel metabolic cages; the urine fluoride content was measured in each group; the urine fluoride content in the fluoride removal group and defluorination control group were measured by the same method at weeks 16 , 20 and 24.

\section{Sample collection and preparation}

The animals in the high fluoride group and high fluoride control group were sacrificed by the cervical dislocation method at week 12 . Cervical spinal cords were taken for flow cytometry observation; another part of the cervical spinal cords was fixed by glutaraldehyde for electron microscopy observation; and another part was fixed by paraformaldehyde, and prepared as paraffin sections for immunohistochemical staining. In addition, the western blot method was used to measure the content of MMP-9 and p53 protein in thoracic spinal cord. The Evans blue (EB) method was used for quantitative analysis of the destruction of the blood-brain barrier. The same procedures were performed in the fluoride removal group and defluorination control group at week 24 for further analysis.

\section{Observation of pathological conditions}

To investigate the ultrastructural changes of the blood-spinal cord barrier, specimens were fixed in $2 \%$ glutaraldehyde, $1 \%$ osmium tetroxide, dehydrated with gradient acetone; embedded in epoxy resin, and prepared as ultrathin sections. The sections were stained by uranyl acetate and lead citrate, then observed under a transmission HITACHI-7500 (Hitachi; Tokyo, JPN) electron microscope. In addition, 5 areas of prepared section were randomly selected for image analysis under a 400 $\times$ microscope. Pathological conditions were graded with Foster's pathological standard designed for neonatal pigs [21], the degree of damage was observed and the final score of the individual areas were scored by a blinded observer: the vascular endothelial cell cavity was recorded as being from well-structured (10 points) to seriously damaged (0 points); the shape of vascular endothelial cells was recorded as being from uniform (10 points) to misshaped (0 points); the basement membrane was recorded as complete (10 points) and continuous (10 points); the astrocyte foot processes were recorded as having 10 points if they had no edema.

\section{Flow cytometry}

Cell apoptosis was detected by flow cytometry. The spinal cord was processed to small fragments; digested by trypsin $(2.5 \mathrm{~g} / \mathrm{l})$ for $10 \mathrm{~min}$; filtered by 200 mesh sieve; centrifuged at $1000 \mathrm{r} / \mathrm{min}$ for 5 min; DMEM/F12 culture medium containing $10 \%$ (volume fraction) fetal bovine serum (GIBCO, Carlsbad, CA, USA) was used for cell suspension; $1000 \mu \mathrm{l}$ of cells with a concentration of $1 \times 10^{6} / \mathrm{ml}$ were added in the wells covered with poly lysine of a 24 -well plate; cells were inoculated in a $37^{\circ} \mathrm{C}$ incubator containing $5 \% \mathrm{CO}_{2}$; the cell culture me- 
dium was replaced every $24 \mathrm{~h}$. After being cultured for 4-5 day, adherent cells were digested by trypsin (2.5 g/l); Annexin V FITC/PI double staining was performed according to the kit's instructions for cells; the cell apoptosis was detected by flow cytometry (Becton-Dickinson FACSCalibur flow cytometer, BD Biosciences, Franklin Lakes, NJ, USA).

\section{Evans blue method for quantitative analysis of blood-brain barrier damage}

Ten rats in the high fluoride group and high fluoride control group were randomly selected and sacrificed at week 12 . $2 \%$ EB ( $3 \mathrm{ml} / \mathrm{kg})$ was intravenously injected; physiological saline was perfused through the left ventricle until the clear liquid flowed out the right atrial appendage. $0.2 \mathrm{~g}$ of spinal cord was taken out, $3 \mathrm{ml}$ dimethylformamide was added and cultured in a water bath at $37^{\circ} \mathrm{C}$ for $8 \mathrm{~h}$; centrifuged for $10 \mathrm{~min}$ with $1500 \mathrm{rpm}$; the supernatant was used to determine the maximum absorption spectra at $632 \mathrm{~nm}$ of EB in a spectrophotometer. The EB content was determined from the standard curve, and the results were recorded as $\mu \mathrm{g} / \mathrm{g}$ (spinal cord). The quantitative analysis was performed in the fluoride removal group and the defluorination control group at week 24 also.

\section{Immunohistochemical analysis}

Routine immunohistochemical staining (SP method) was used in the current study. The primary antibody for MMP-9 was rabbit polyclonal to MMP-9 antibody (ab38898, Abcam plc, Cambridge, MA, USA); and rabbit polyclonal to p53 antibody for p53 primary antibody (ab1431, Abcam plc); the negative control antibody was PBS (ab154878, Abcam plc); the remaining steps were the same as described before. The cells having brown yellow grain coarse particle distribution or brown yellow fine particle distribution were defined as positive. The optical density of the positive reaction was determined by the TONGJ HPIAS-1000 color pathological image analysis system (Qingping image technology Ltd., Wuhan, CHN).

\section{Western blot analysis}

The spinal cord tissue was cut into the cytoplasmic extract (100 g/l), smashed by the ultrasonic tissue crushing apparatus, lysed in ice for 30 min then centrifuged at $10000 \mathrm{r} / \mathrm{min}$ for $15 \mathrm{~min}$. The supernatant was extracted and the protein concentration was measured by a Coomassie brilliant blue G-250 kit. The total protein was transferred to PVDF membrane (EMD Millipore, Billerica, MA, USA) through $12 \%$ sodium dodecyl sulfate-polyacrylamide gel electrophoresis (SDS-PAGE), followed by blocking with $5 \%$ skim milk for $1 \mathrm{~h}$.
The rabbit polyclonal anti-MMP-9 antibody (1: 500) and rabbit polyclonal anti-p53 antibody (1 : 500) were added and cultured at $4^{\circ} \mathrm{C}$ overnight; the normal goat IgG-HRP (1: 5000, sc-2741 Santa Cruz Biotech, Autogenbioclear; Calne, UK) was added and incubated at $37^{\circ} \mathrm{C}$ for $45 \mathrm{~min}$. An imaging system (Odyssey, LI-COR Biosciences, Lincoln, NE, USA) was used for semi-quantitative analysis. $\beta$-actin served as a control.

\section{Statistical analysis}

SPSS 18.0 statistical software (IBM, Chicago, IL, USA) was used to analyze all the data. The results were presented as mean \pm SD. Fisher's exact test and test were used for comparison between groups. $P<0.05$ was considered as statistically significant.

\section{Results}

After drinking fluoridated water for 12 weeks, the incisors of rats in the high fluoride group all presented incisor enamel, while the incisors were orange translucent in the high fluoride control
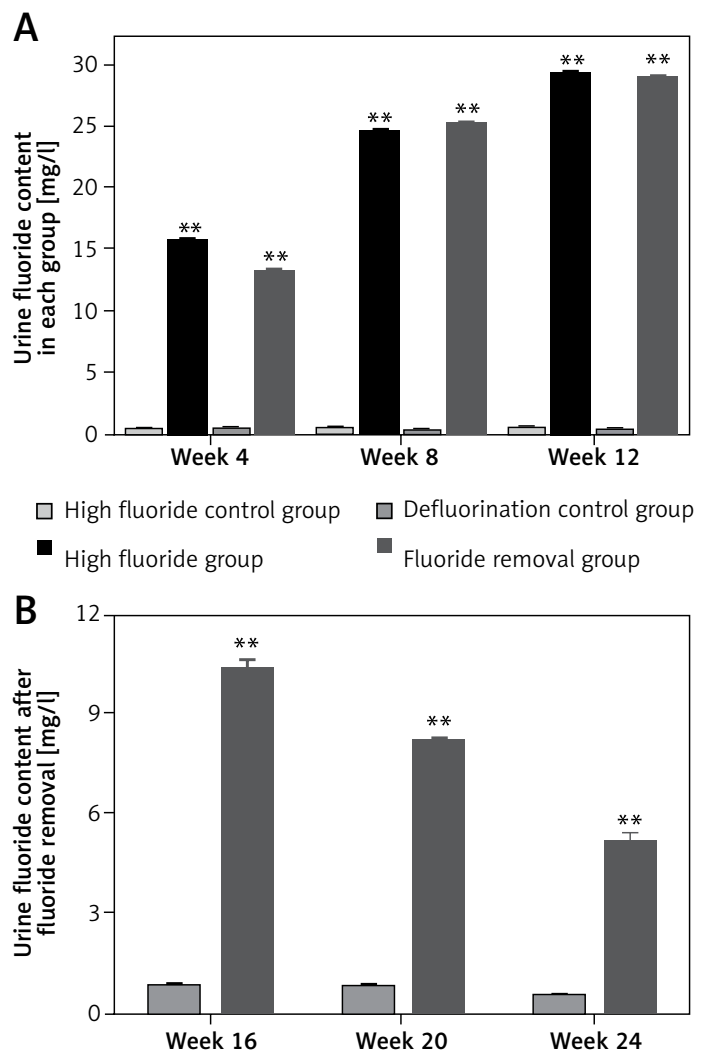

Figure 1. Urine fluoride content in each group. A - The urine fluoride content in rats drinking fluoridated water continually increased from week 4 to week 12. B - The urine fluoride content was still significantly higher in the fluoride removal group than the defluorination control group from week 16 to week 24

${ }^{* *} p<0.01$, compared with high fluoride control group and defluorination control group. 

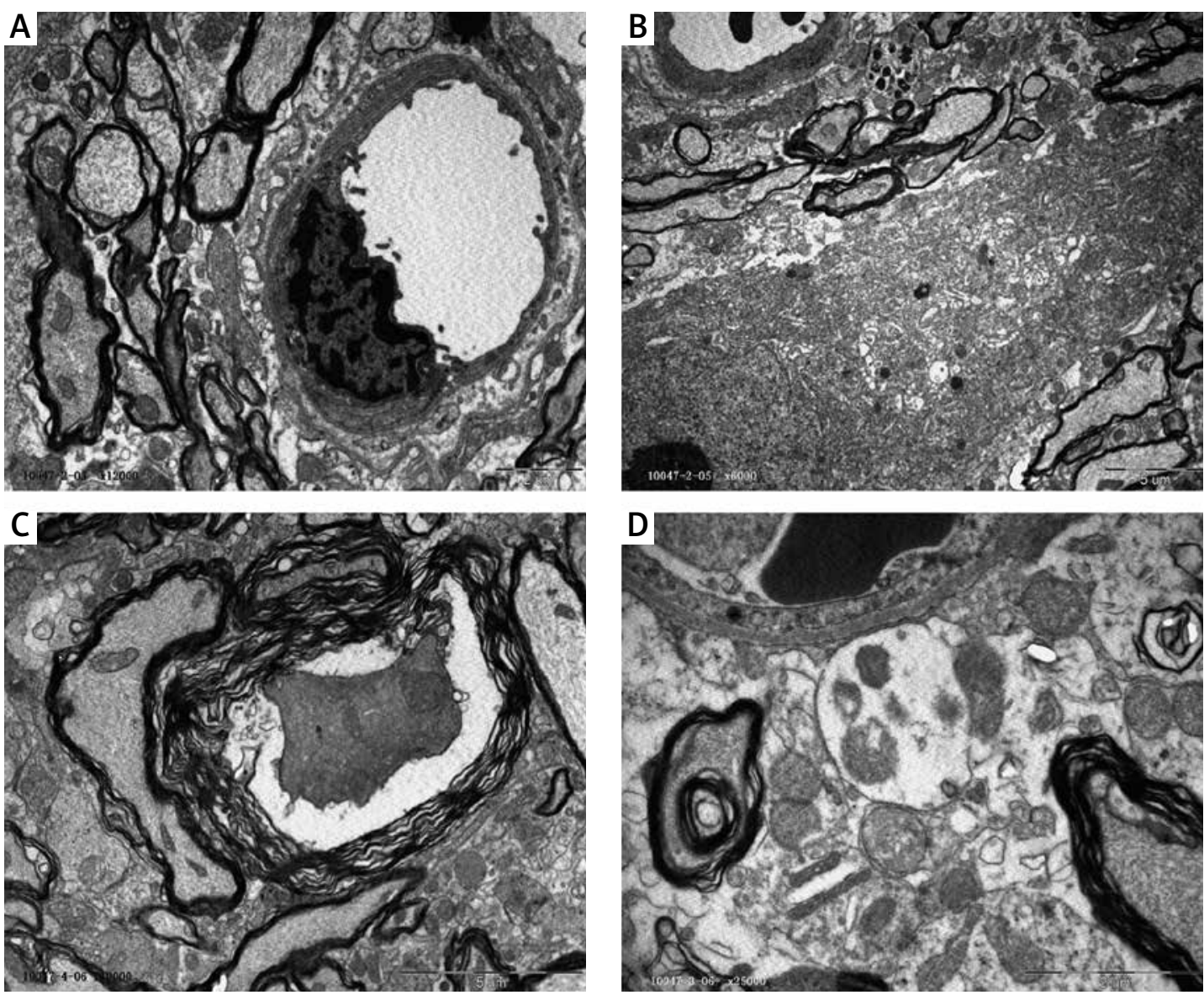

E

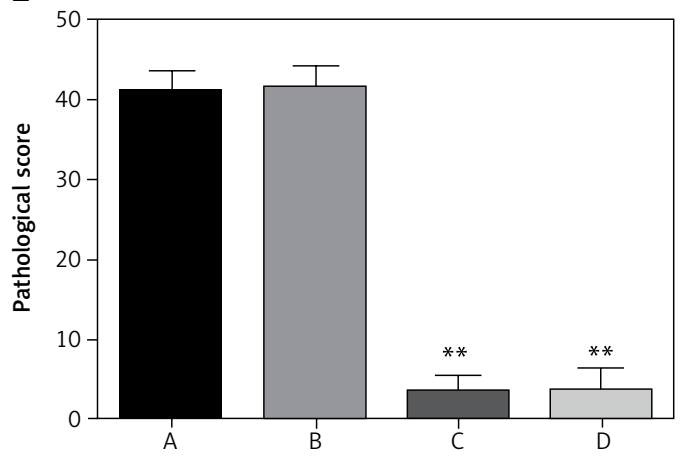

- High fluoride control group $\quad \square$ High fluoride group

$\square$ Defluorination control group $\square$ Fluoride removal group

group, which suggests that rats in the high fluoride group had chronic fluorosis.

\section{Urine fluoride content in each group}

The urine fluoride content in rats drinking fluoridated water continually increased from week 4 to week 12 (Figure $1 \mathrm{~A}$ ); the urine fluoride content in rats drinking fluoridated water was significantly higher in weeks 4,8 and 12 compared with rats that did not drink fluoridated water $(p<0.01)$. In addition, after removal of fluoridated water, despite decreased urine fluoride content in the fluoride removal group (Figure $1 \mathrm{~B}$ ),

Figure 2. Electron microscope images in each group. A - High fluoride control group (12 000x), B - defluorination control group (6000x), C - high fluoride group (12 000x), D - fluoride removal group (2500x), E - the pathological conditions were compared with pathological scores in each group

the urine fluoride content was still significantly higher when compared with the defluorination control group $(p<0.01)$. Even 3 months after the removal of fluoridated water, the urine fluoride content was still significantly higher $(p<0.01$ compared with the defluorination control group, Figure $1 \mathrm{~B}$ ).

\section{Pathological conditions}

Observation results showed that the blood-spinal cord barrier was intact in the high fluoride control group and defluorination control group (Figures $2 \mathrm{~A}, \mathrm{~B}$ ); their vascular endothelial cell cav- 
A

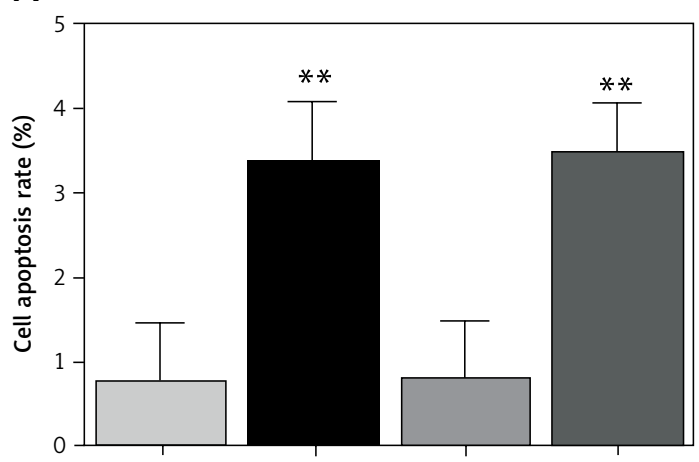

C

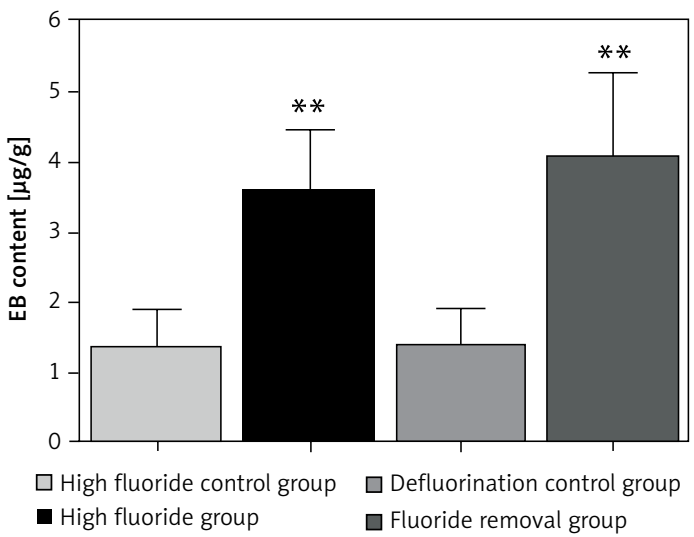

B
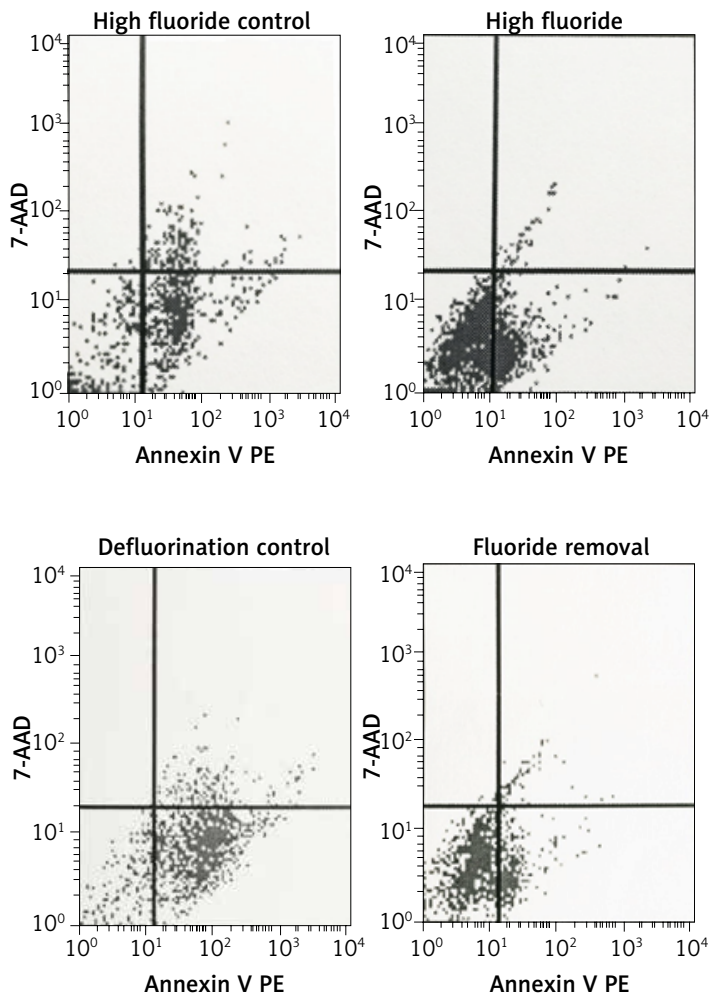

Figure 3. Comparison of cell apoptosis rate and Evans blue (EB) content in spinal cord of rats in each group. $\mathbf{A}-$ Cell apoptosis rate, $\mathbf{B}$ - flow cytometry picture in each group, $\mathbf{C}-\mathrm{EB}$ content

ity was smooth and well structured; the basement membrane was complete and continuous; their astrocyte foot processes had no edema. In contrast, the gap height of the blood-spinal cord barrier in rats of the high fluoride group (Figure $2 \mathrm{C}$ ) expanded; edema could be observed in the astrocyte cell foot; the intracellular components disappeared and were replaced by free water space; most microfilaments in the foot process were degraded; mitochondria were degenerated; the electron density of matrix increased; cystic cell expansion, partial membrane dissolution and rupture could be observed; the microvascular endothelial cells in the blood-brain barrier were darkened; mitochondria and rough endoplasmic reticulum were degenerated; endothelial cell dysfunction occurred; the microvascular free surface had denatured small microvilli and edema. We still could observe edema in astrocyte cells around the blood-brain barrier of rats in the fluoride removal group (Figure $2 \mathrm{D}$ ), a small part of glial cells had obvious foot process edema and an edema sheath formed around the blood vessels; partial endothelial cell dysfunction occurred, as well as mild extension of the nucleus and cystic cell edema; a small piece of water gap existed in the nerve felt, which indicated that ultrastructural damage occurred in the spinal cord of fluorosis rats and the damage did not improve after removal of fluoride in the short term (12 weeks). The average pathological score in the high fluoride control group was $41.35 \pm 2.51$, which is significantly higher than the fluoride group and fluoride removal group $(p<0.01$, Figure $2 \mathrm{E}$ ).

\section{Cell apoptosis detected by flow cytometry}

Compared with the high fluoride control group, the spinal cord cell apoptosis rate in the high fluoride group increased significantly $(p<0.01$, Figures 3 A, B). However, removal of fluoride in the short term did not attenuate the cell apoptosis rate. The cell apoptosis rate in the spinal cord of the high fluoride group and fluoride removal group was significantly higher than in the high fluoride control group and defluorination control group ( $p<0.01$, Figures $3 \mathrm{~A}, \mathrm{~B})$.

\section{Evans blue content in spinal cord tissues}

Compared with the high fluoride control group, the EB content in the spinal cord of the high fluoride group increased significantly ( $p<0.01$, Figure $3 \mathrm{C}$ ). Removal of fluoride in the short term did not decrease EB content. The EB content in the spinal 
MMP-9

A

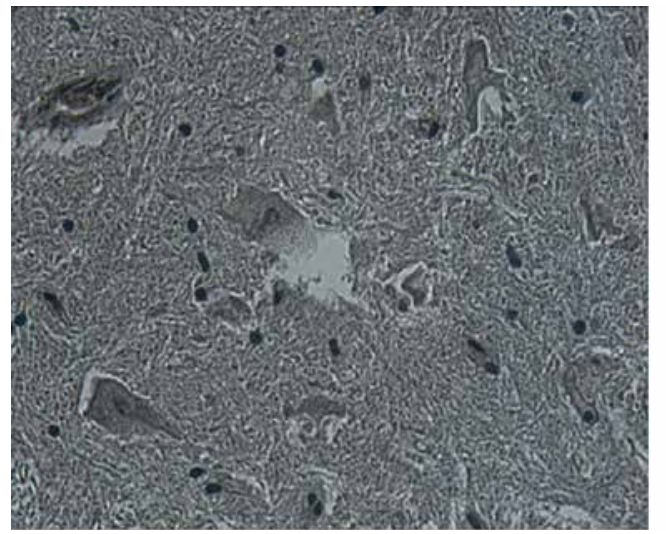

B

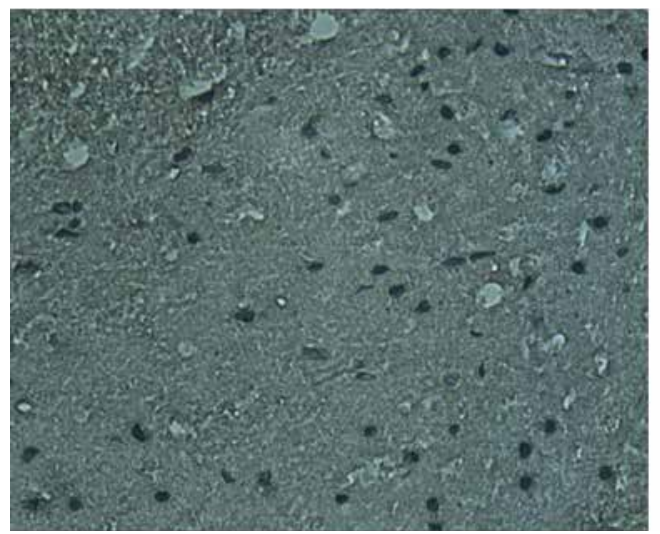

C

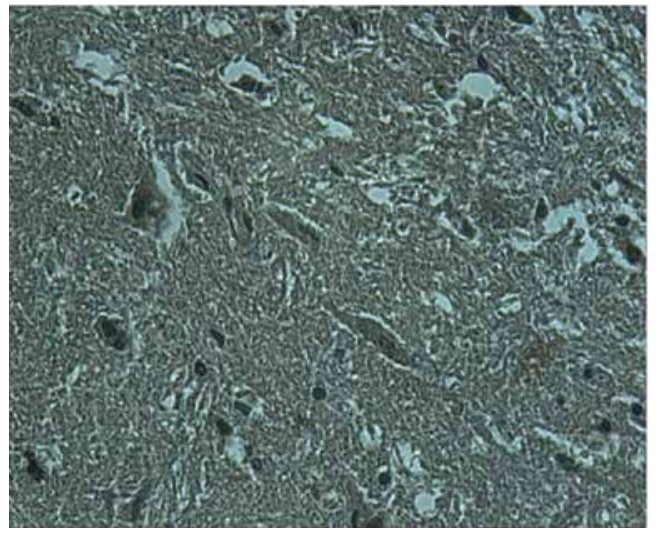

D

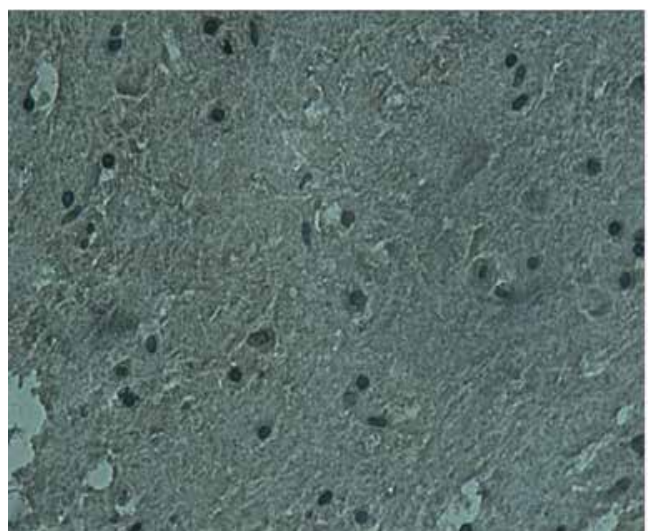

p53
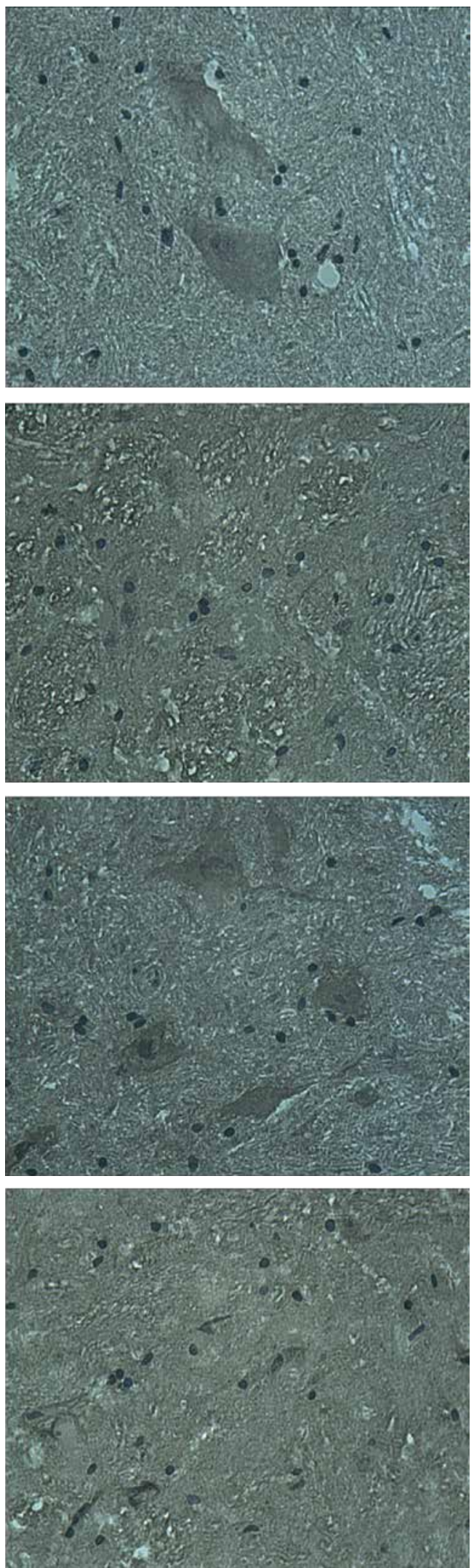

Figure 4. Immunohistochemistry results of MMP-9 and p53 expression. A - High fluoride group (400x), B - high fluoride control group (200x), C - fluoride removal group (400x), D - defluorination control group (200x) 
E

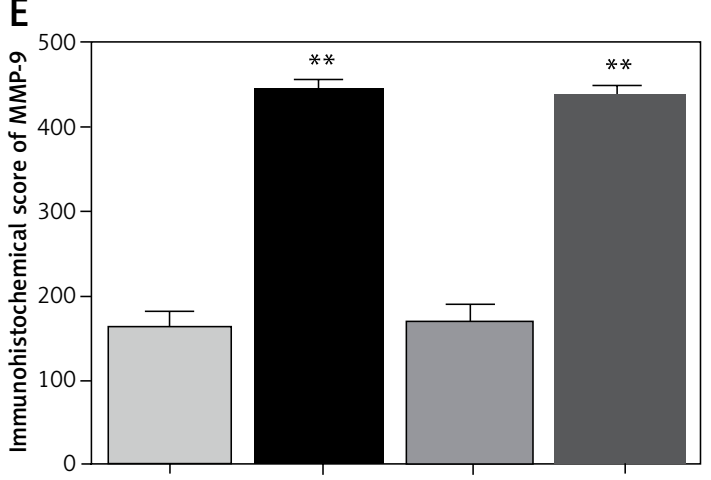

$\square$ High fluoride group $\quad \square$ High fluoride group

- High fluoride control group Defluorination control group

Figure 4. Cont. E-Immunohistochemical score comp score comparison of p53 in each group

${ }^{* *} p<0.01$, compared with high fluoride control group and defluorination control group.

cord of the high fluoride group and fluoride removal group was significantly higher than that in the high fluoride control group and defluorination control group ( $p<0.01$, Figure $3 \mathrm{C}$ ). The results showed that the high fluoride group had increased permeability of the blood-spinal cord barrier in rats; and defluorination had almost no recovery effect.

\section{Immunohistochemistry results}

The cytoplasm of positively expressed MMP-9 and $p 53$ cells was brown. Brownish yellow could be observed in cell cytoplasm of the high fluoride group (Figure $4 \mathrm{~A}$ ), which could occasionally be observed in control groups (Figure $4 \mathrm{~B}$ ); karyopyknosis and perinuclear vacuolization could be observed in partial cells, which mainly existed in the gray matter neurons, glial cells and microvascular endothelial cells; the integral optical density in the high fluoride group was significant higher compared with the high fluoride control group. Positively expressed gray matter neurons, glial cells and microvascular endothelial cells could also be observed in the fluoride removal group (Figure $4 \mathrm{C}$ ); the integral optical density of the fluoride removal group was significantly different compared to the defluorination control group (Figure $4 \mathrm{D}$ ) but had no significant difference compared with the high fluoride group $(p>0.05)$ (Figures $4 \mathrm{E}, \mathrm{F})$.

\section{Western blot results}

The optical density measurement results of western blot showed that the expression levels of MMP-9 and p53 (Figure 5 A) increased significantly in the high fluoride group compared with the control group ( $p<0.01)$; after the removal of fluoridated water, the expression levels of MMP-9 and p53 were still significantly higher (Figure $5 \mathrm{~B}, p<0.01$ compared with the defluorination control group).

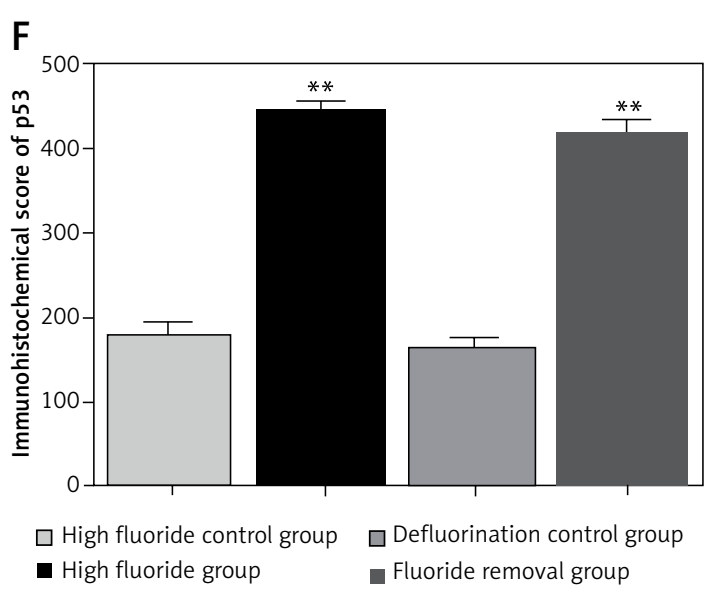

$\begin{array}{ll}\square \text { High fluoride group } & \square \text { Defluorination control group removal group }\end{array}$

son of MMP-9 in each group, F - immunohistochemical Discussion

Fluorosis can cause spinal cord injury, but the related mechanism is still not clear [22]. There have been some reports about damage caused by high fluoride levels to the blood-brain barrier in recent years, but most of the research focused on changes of nerve cells in the brain [23-25]. Closely connected vascular endothelial cells, basement membrane and the astrocytes outside of the basement membrane are important morphological bases of the blood-brain barrier. In our research, the flow cytometry results showed that the apoptosis of nerve cells in the spinal cord of the high fluoride group was significantly higher than that of the control rats; this result further confirmed that chronic fluorosis induced apoptosis of spinal cord nerve cells. The electron microscopy directly demonstrated the microstructure of the blood-brain barrier. The astrocyte edema, mitochondrial degeneration, and microvascular endothelial cell dysfunction showed the pathological damage of chronic fluorosis. The quantitative analysis of EB indicated that the blood-brain barrier function of the spinal cord was damaged severely after fluorosis. The apoptosis of neural cells is the direct consequence of fluoride exposure [26]. There are many mechanisms involved in the apoptosis of neural cells. However, the study on the neuronal apoptosis induced by fluorosis is still in the exploratory stage at present.

MMP-9 is a member of the MMP family. The substrates of MMP-9 are mainly gelatin, elastin, fibronectin and collagen type VI, which is the main component of basement membrane; the main role of MMP-9 is degradation of basement membrane in pathological conditions in vivo [27]. The MMP family is involved in multiple pathological processes including sclerosis, brain trauma, spinal cord damage, meningitis, cerebral vascular disease and central nervous system disease [28, 29]. 
A $\begin{array}{llllllllllllllll}\mathrm{F} 1 & \mathrm{~F} 2 & \mathrm{~F} 3 & \mathrm{~F} 4 & \mathrm{FC} 1 & \mathrm{FC} 2 & \mathrm{FC} 3 & \mathrm{FC} 4 & \mathrm{D} 1 & \mathrm{D} 2 & \mathrm{D} 3 & \mathrm{D} 4 & \mathrm{DC} 1 & \mathrm{DC} 2 & \mathrm{DC} & \mathrm{DC} 4\end{array}$ MMP-9 p53

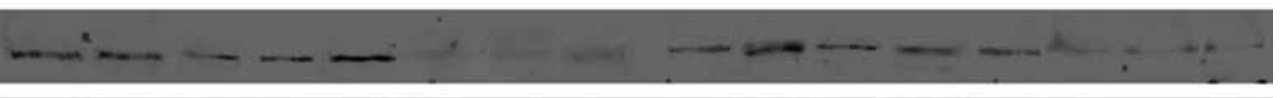

$\beta$-actin

B

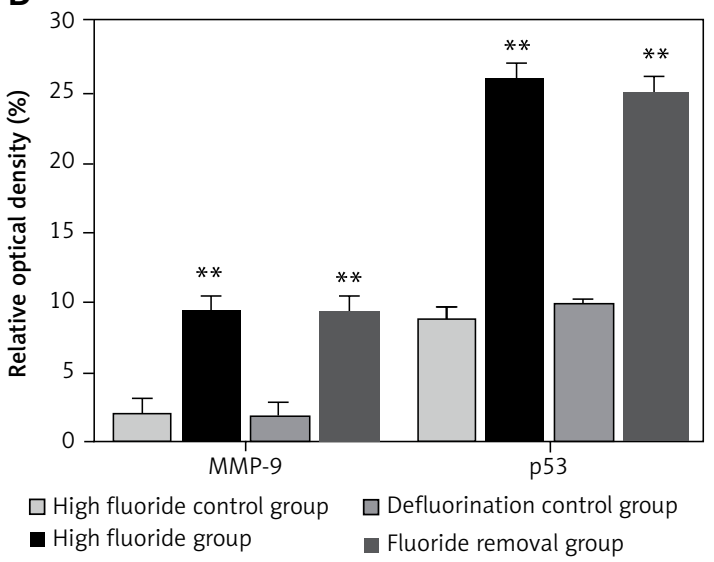

In normal brain tissues, the expression of MMP-9 is low. Martins et al. [19] found that the increased expression of MMP-9 can increase the permeability of the blood-brain barrier. Highly expressed MMP-9 is also confirmed to be correlated with multiple nerve injury [30, 31]. Extracellular matrix (ECM) between the vascular basement membrane and mesenchyme is the key structural foundation to maintain the integrity of the blood-brain barrier. The main components of ECM such as collagen, glycoprotein and lipoprotein are substrates of MMP-9 [32, 33]. Through degradation of ECM, MMP-9 makes the blood-brain barrier open and recruit lymphocytes, thus resulting in multiple pathological processes in the central nervous system [34]. Takata et al. [35] indicated that TNF- $\alpha$ can stimulate the release of MMP-9 by the cell cycle of the blood-brain barrier, which leads to damage of the blood-brain barrier. Hong et al. [36] indicated that the expression of MMP-9 is regulated by various growth factors and cytokines, which can be activated by activating the MAPK signaling pathway. As for the further mechanism (signal channel) of MMP-9 in blood-brain barrier damage caused by fluorosis, it is very necessary to study the related mechanism in the next step.

Apoptosis is controlled by genes. p53 is one of the main gene regulating signal transduction of cell apoptosis [20]. Inducing apoptosis of cells is one of the basic functions of $\mathrm{p} 53$. $\mathrm{p} 53$ is a $20 \mathrm{~kb}$ fulllength gene located on the human 17 p13.1 gene;
Figure 5. Western blot results of expression of MMP-9 and p53. A - Western blot images, $\mathbf{B}$ - relative optical density measurement results. F1-F5: high fluoride group, FC1-FC3: high fluoride control group, D1-D5: fluoride removal group, DC1-DC4: defluorination control group

composed of 11 exons and 10 introns. The promoter of $\mathrm{p} 53$ does not contain common start sequence of CAAT box, TATA and GC box. As a transcription factor of apoptosis, activated p53 can effectively induce cell apoptosis through the death receptor pathway and mitochondrial pathway [37]. The P53 gene can be divided into wild type wtp53 and mutant type mtP53: wtp53 promotes apoptosis and mtP53 inhibit apoptosis. Wtp53 is also a negative regulator of cell growth. Increased expression of wtp53 induced the expression of related genes and inhibited the growth and proliferation of injured cells; thus it made DNA repair damage or start apoptosis when severely damaged. The chronic injury of nervous tissue leads to the increased expression of p53; brain damage can also lead to the increasing protein expression of wtp53 [38].

The potential process of p53 mediated apoptosis in neural cells includes the following: 1) high p53 expression is induced by some injury factors; 2) p53 protein accumulates in the nucleus, combines with damaged DNA, then is activated, regulates expression of related gene and inhibits the growth and proliferation of cells; 3) the expression of $p 53$ protein selectively inhibit the transcription of c-myc gene, thus inducing the cell apoptosis; 4) p53 increases the expression of Bax and IGF, and reduces the expression of antiapoptotic protein bcl-2; 5) p53 may also directly down-regulate the expression of $\mathrm{BCl}-2$, thereby increasing the susceptibility of neuronal apopto- 
sis [32]; 6) p53 protein can form protein complexes with other proteins such as replication protein antigen (RPA), transcription factor complex IIH (TFIIH), repair factor GADD-45, etc., and participate in the synthesis, replication, repair and damage of DNA [34]. Our research also showed that chronic fluorosis can induce expression of p53 protein; highly expressed p53 is roughly matched with apoptosis of neural cells. These results suggest that p53 protein is involved in the physiological apoptosis process of spinal nerve cells caused by fluorosis.

The relationship between p53 gene and MMP 9 has attracted the attention of researchers. Franchi et al. [39] indicated that mutation of p53 can up-regulate MMP-9 transcription through the NOS and EGFR pathway. Ma et al. [40] also found that expression of MMP-9 was positively correlated with p53 expression in human meningioma tissue. Consistent with our research, these studies suggest that p53 and MMP-9 play synergic roles in the development of nervous system lesions.

For fluorosis caused by drinking water, the removal of the cause is an effective method to control chronic fluorosis. If the fluoride intake is reduced, the accumulated fluoride could be excreted gradually through a physiological metabolism, but the reverse recovery of the damaged organism is very difficult. Huang's research [41] showed that 40 years after a change of drinking water in a high fluoride area, the bone fluorosis rehabilitation of fluoride poisoned residents was quite limited, and the rehabilitation of the central nervous system was slower. Our results showed that the spinal cord injury in rat blood-brain barrier had no obvious recovery after short-term defluorination. These results indicate that once the chronic fluorosis has occurred, although the consumption of fluoride has stopped, the apoptosis of spinal cord nerve cells is still unable to recover in a relatively short period. This may be related to the increased expression of MMP-9 and p53. However, there are multiple mechanisms involved in apoptosis of nerve cells. The regulation mechanism of the blood-brain barrier has not been fully elucidated. At the same time, we did not carry out related research on the signal transduction pathway of spinal cord nerve cell apoptosis and bloodbrain barrier injury caused by fluorosis. Research on fluorosis poisoning causing apoptosis is still in the exploration stage. Our study only referenced partial research about brain nerve injury. We are exploring other possible mechanisms now. For the recovery of neural injury after defluorination, the current study only evaluated medium and short-term results, and the long-term defluorination results (over 12 months) are being observed.

In conclusion, our research further confirmed that chronic fluorosis can lead to apoptosis of spinal neurons in rats. After the short-term remov- al of fluoride, the apoptosis of the spinal cord could not be restored. The increased expression of MMP-9 and p53 might be involved in the underlying mechanism.

\section{Conflict of interest}

The authors declare no conflict of interest.

\section{Reference}

1. Yamamoto G, Yoshitake K, Sato T, Kimura T, Ando T. Distribution and forms of fluorine in whole blood of human male. Anal Biochem 1989; 182: 371-6.

2. Clifford H, Olszowy H, Young M, Hegarty J, Cross M. Fluoride content of powdered infant formula meets Australian Food Safety Standards. Aust N Z J Public Health 2009; 33: 573-6.

3. Singh N, Verma KG, Verma P, Sidhu GK, Sachdeva S. A comparative study of fluoride ingestion levels, serum thyroid hormone \& TSH level derangements, dental fluorosis status among school children from endemic and non-endemic fluorosis areas. Springerplus 2014; 3: 7.

4. Abdel-Gawad FA, Ashmawy MH, Zaki SM, Abdel-Fatah GH. Lung damage after long-term exposure of adult rats to sodium fluoride. Arch Med Sci 2014; 10: 1035-40.

5. Adali MK, Varol E, Aksoy F, et al. Impaired heart rate recovery in patients with endemic fluorosis. Biol Trace Elem Res 2013; 152: 310-5.

6. Zhang J, Zhu WJ, Xu XH, Zhang ZG. Effect of fluoride on calcium ion concentration and expression of nuclear transcription factor kappa-B rho65 in rat hippocampus. Exp Toxicol Pathol 2011; 63: 407-11.

7. Li M, Gao Y, Cui J, et al. Cognitive impairment and risk factors in elderly people living in fluorosis areas in China. Biol Trace Elem Res 2016; 172: 53-60.

8. Song GH, Gao JP, Wang CF, et al. Sodium fluoride induces apoptosis in the kidney of rats through caspase-mediated pathways and DNA damage. J Physiol Biochem 2014; 70: 857-68.

9. Haimanot RT. Neurological complications of endemic skeletal fluorosis, with special emphasis on radiculomyelopathy. Paraplegia 1990; 28: 244-51.

10. Choi AL, Zhang Y, Sun G, et al. Comment on "Severe dental fluorosis and cognitive deficits". Neurotoxicol Teratol 2015; 50: 32.

11. Chakraborti D, Rahman MM, Chatterjee A, et al. Fate of over 480 million inhabitants living in arsenic and fluoride endemic Indian districts: magnitude, health, socio-economic effects and mitigation approaches. J Trace Elem Med Biol 2016; 38: 33-45.

12. Richter H, Kierdorf U, Richards A, Kierdorf H. Dentin abnormalities in cheek teeth of wild red deer and roe deer from a fluoride-polluted area in Central Europe. Ann Anat 2010; 192: 86-95.

13. Khan SA, Singh RK, Navit S, et al. Relationship between dental fluorosis and intelligence quotient of school going children in and around lucknow district: a cross-sectional study. I Clin Diagn Res 2015; 9: ZC10-5.

14. Das K, Mondal NK. Dental fluorosis and urinary fluoride concentration as a reflection of fluoride exposure and its impact on IQ level and BMI of children of Laxmisagar, Simlapal Block of Bankura District, W.B., India. Environ Monit Assess 2016; 188: 218.

15. Sun Z, Liu F, Yang H, et al. Observations of the supra-micro-structure of the brain hippocampus in mice exposed 
to high fluoride in drinking water. Chin J Endemiol 2000; 19: 78-81.

16. Mohamed NE. Erratum to: The role of calcium in ameliorating the oxidative stress of fluoride in rats. Biol Trace Elem Res 2016; 170: 145

17. Yadav SS, Kumar R, Khare P, Tripathi M. Oxidative stress biomarkers in the freshwater fish, heteropneustes fossilis (Bloch) exposed to sodium fluoride: antioxidant defense and role of ascorbic acid. Toxicol Int 2015; 22: 71-6.

18. Dittrich S, Sunyakumthorn P, Rattanavong S, et al. Blood brain barrier function and biomarkers of central nervous system injury in rickettsial versus other neurological infections in Laos. Am J Trop Med Hyg 2015; 93: 232-7.

19. Martins T, Baptista S, Goncalves J, et al. Methamphetamine transiently increases the blood-brain barrier permeability in the hippocampus: role of tight junction proteins and matrix metalloproteinase-9. Brain Res 2011; 1411: 28-40.

20. Chiu TH, Lan KY, Yang MD, et al. Diallyl sulfide promotes cell-cycle arrest through the p53 expression and triggers induction of apoptosis via caspase- and mitochondria-dependent signaling pathways in human cervical cancer Ca Ski cells. Nutr Cancer 2013; 65: 505-14.

21. Foster KA, Colditz PB, Lingwood BE, Burke C, Dunster KR, Roberts MS. An improved survival model of hypoxia/ ischaemia in the piglet suitable for neuroprotection studies. Brain Res 2001; 919: 122-31.

22. Kurdi MS. Chronic fluorosis: the disease and its anaesthetic implications. Indian J Anaesth 2016; 60: 157-62.

23. Lou DD, Guan ZZ, Liu YJ, et al. The influence of chronic fluorosis on mitochondrial dynamics morphology and distribution in cortical neurons of the rat brain. Arch Toxicol 2013; 87: 449-57.

24. Zhang KL, Lou DD, Guan ZZ. Activation of the AGE/RAGE system in the brains of rats and in SH-SY5Y cells exposed to high level of fluoride might connect to oxidative stress. Neurotoxicol Teratol 2015; 48: 49-55.

25. Ma J, Liu F, Liu P, et al. Impact of early developmental fluoride exposure on the peripheral pain sensitivity in mice. Int J Dev Neurosci 2015; 47: 165-71.

26. Ke L, Zheng X, Sun Y, Ouyang W, Zhang Z. Effects of sodium fluoride on lipid peroxidation and PARP, XBP-1 expression in PC12 cell. Biol Trace Elem Res 2016; 173: 161-7.

27. Ugarte-Berzal E, Vandooren J, Bailon E, Opdenakker G, Garcia-Pardo A. Inhibition of MMP-9-dependent degradation of gelatin, but not other MMP-9 substrates, by the MMP-9 hemopexin domain blades 1 and 4.J Biol Chem 2016; 291: 11751-60.

28. Chen J, Luo M, Wang W, et al. Altered proteolytic activity and expression of MMPs and aggrecanases and their inhibitors in Kashin-Beck disease. J Orthop Res 2015; 33: 47-55.

29. Zwierzchowski TJ, Stasikowska-Kanicka O, Janus J, Konecki W, Danilewicz M, Fabis J. Assessment of apoptosis, MMP-1, MMP-3, TIMP-2 expression and mechanical and biochemical properties of fresh rabbit's medial meniscus stored two weeks under tissue culture conditions. Arch Med Sci 2014; 10: 167-73.

30. Hou H, Zhang G, Wang H, Gong H, Wang C, Zhang X High matrix metalloproteinase- 9 expression induces angiogenesis and basement membrane degradation in stroke-prone spontaneously hypertensive rats after cerebral infarction. Neural Regen Res 2014; 9: 1154-62.

31. Miyazaki K, Ohta Y, Nagai M, et al. Disruption of neurovascular unit prior to motor neuron degeneration in amyotrophic lateral sclerosis. J Neurosci Res 2011; 89: 718-28

32. Tsuji K, Aoki T, Tejima E, et al. Tissue plasminogen activator promotes matrix metalloproteinase-9 upregulation after focal cerebral ischemia. Stroke 2005; 36: 1954-9.

33. Vitlianova K, Georgieva J, Milanova M, Tzonev S. Blood pressure control predicts plasma matrix metalloproteinase-9 in diabetes mellitus type II. Arch Med Sci 2015 11: 85-91.

34. Stein VM, Puff C, Genini S, Contioso VB, Baumgartner $W$, Tipold $A$. Variations on brain microglial gene expression of MMPS, RECK, and TIMPs in inflammatory and non-inflammatory diseases in dogs. Vet Immunol Immunopathol 2011; 144: 17-26

35. Takata F, Dohgu S, Matsumoto J, et al. Brain pericytes among cells constituting the blood-brain barrier are highly sensitive to tumor necrosis factor-alpha, releasing matrix metalloproteinase- 9 and migrating in vitro. Neuroinflammation 2011; 8: 106

36. Hong S, Park KK, Magae J, et al. Ascochlorin inhibits matrix metalloproteinase-9 expression by suppressing activator protein-1-mediated gene expression through the ERK1/2 signaling pathway: inhibitory effects of ascochlorin on the invasion of renal carcinoma cells. J Biol Chem 2005; 280: 25202-9.

37. Seth R, Yang C, Kaushal V, Shah SV, Kaushal GP. p53-dependent caspase-2 activation in mitochondrial release of apoptosis-inducing factor and its role in renal tubular epithelial cell injury. J Biol Chem 2005; 280: 31230-9.

38. Gidday JM, Gasche YG, Copin JC, et al. Leukocyte-derived matrix metalloproteinase- 9 mediates blood-brain barrier breakdown and is proinflammatory after transient focal cerebral ischemia. Am J Physiol 2005; 289: H558-68.

39. Franchi A, Santucci M, Masini E, Sardi I, Paglierani M, Gallo O. Expression of matrix metalloproteinase 1, matrix metalloproteinase 2, and matrix metalloproteinase 9 in carcinoma of the head and neck. Cancer 2002; 95: 1902-10.

40. Ma C, Xu G. Expression of MMP-9 and p53 in meningiomas tissues. J Zhengzhou Univ 2004; 39: 616-9.

41. Huang CQ. X-ray signs of bone and joint among residents of endemic fluorosis area 40 years after improvement of water. Chin J Endemiol 2006; 25: 192-5. 